\title{
Clinical Outcomes of Successful Revascularization of Chronic Total Coronary Occlusions with Bioresorbable Vascular Scaffolds: A Systematic Review
}

\author{
Roman Marchenko $^{1}$, Salik Nazir ${ }^{1}$, Shelina Malla ${ }^{1}$, Anthony Donato ${ }^{1}$ \\ 1. Internal Medicine, Reading Hospital-Tower Health, Reading, USA
}

Corresponding author: Roman Marchenko,dr.rmarch@gmail.com

\begin{abstract}
Revascularization of chronic total occlusions (СTO) with percutaneous coronary intervention is associated with favorable long-term clinical and echocardiographic outcomes. Whether bioresorbable vascular scaffolds (BVS) would be advantageous in the treatment of CTO is unknown as patients with these lesions were generally excluded from large BVS randomized trials. We performed a systematic review, which sought to summarize known data on mid- to long-term clinical outcomes for BVS in CTO. We searched MEDLINE, EMBASE, clinicaltrials.gov, and the Cochrane Library through April 2018 to look for studies on implantation of BVS in CTO. Outcomes of interest included myocardial infarction, cardiac death, all-cause mortality, major adverse cardiac events (MACE), vessel restenosis, scaffold thrombosis, and target lesion revascularization. A total of 13 articles met the inclusion criteria for analysis. All studies were observational with a total number of patients of 1,077. Only two studies included comparator groups which retrospectively compared BVS with drug-eluting stents (DES). The studies had variable size (21 to 537) and follow-up duration (3-23 months). The review showed favorable outcomes for BVS implantation in CTO with the reported incidence of MACE ranged from $0 \%$ to $6.7 \%$ with no significant differences between BVS and DES groups in double arm studies. Although data on the use of first-generation BVS in CTO are sporadic and limited by small sample observational studies, available evidence is promising and suggests of acceptable outcomes comparable with second generation DES. Further investigation with randomized clinical trials and use of newer generation scaffolds is required.
\end{abstract}

Received 10/22/2018 Review began 10/31/2018 Review ended 11/23/2018 Published 11/28/2018

๑) Copyright 2018

Marchenko et al. This is an open access article distributed under the terms of the Creative Commons Attribution License CC-BY 3.0., which permits unrestricted use, distribution, and reproduction in any medium, provided the original author and source are credited.
Categories: Cardiology

Keywords: coronary artery disease, percutaneous coronary intervention, chronic total occlusion, bioresorbable vascular scaffold

\section{Introduction And Background}

Chronic total occlusion (CTO) of the coronary artery is defined as a complete vessel occlusion with thrombolysis in myocardial infarction (TIMI) flow grade of 0 lasting for more than three months [1]. CTOs are present in up to $20 \%$ of patients with coronary artery disease undergoing elective angiography [2]. Revascularization of CTO with percutaneous coronary intervention (PCI) is associated with angina relief, improved left ventricular function, reduction in the rate of myocardial infarction, less need for subsequent coronary artery bypass grafting and better patient survival regardless of the presence of collateral circulation [3-5]. However, because multiple sequential long stents are frequently required (termed vessel "caging") to treat a chronically occluded vessel, the vessel is subject to risks of late stent thrombosis and restenosis [6-8]. Bioresorbable vascular scaffolds (BVS) offer an alternative treatment option, since their unique properties allow them to potentially promote vessel healing, permit vascular remodeling, avoid late lumen enlargement, and restore of normal vasomotion, which theoretically avoid the vessel caging issues and risk of late thrombosis $[9,10]$. Whether BVS would be advantageous in treatment of CTO is currently unknown as patients with these lesions were generally excluded from large BVS randomized trials. We performed a systematic review, which sought to summarize known data on mid- to long-term clinical outcomes for BVS in chronic total occlusion.

\section{Review}

\section{Materials and methods}

This review was conducted in accordance with Preferred Reporting Items for Systematic Reviews and MetaAnalyses (PRISMA) guidelines [11]. We performed a systematic electronic search of MEDLINE (via PubMed), EMBASE, clinicaltrials.gov and the Cochrane library for case series, observational studies, clinical trials, and systematic reviews on the Absorb bioresorbable vascular scaffolds (Abbott Vascular, Santa Clara, CA) in chronic total occlusions published from inception until April 26, 2018. Two broad search themes, "bioresorbable vascular scaffold" and "chronic total occlusion" were used (see Appendix). These themes were combined using Boolean operator "AND". We also performed an additional search of references from included articles and articles that cited the included studies to identify additional publications. An 
experienced librarian was involved into the development of search strategy and search process to assure quality. Authors of included publications were contacted via e-mail in cases when additional information was required. Studies were included into review if they fulfilled the following inclusion criteria: 1) included implantation of bioresorbable vascular scaffolds; 2 ) included patients $\geqslant 18$ years of age; 3 ) included patients having chronic total occlusion (CTO) of one or more coronary arteries; 4) reported on at least one of our safety and efficacy outcomes: myocardial infarction (MI), cardiac death, all-cause mortality, major adverse cardiac events (MACE), vessel restenosis, scaffold or stent thrombosis (ST), and target lesion revascularization (TLR); 5) reported at least three months of clinical follow-up data. Criteria for exclusion from the review were: 1) non-English language articles; 2) case reports and case series with fewer than 10 cases; 3) non-human studies. Screening of articles for eligibility and data extraction was performed by two independent reviewers (RM, SN). Any disagreements between reviewers were resolved by consensus using a third reviewer $(\mathrm{AD})$. Extracted data included study type and design, year of publication, demographic and clinical characteristics, primary and secondary outcomes, duration of follow-up. Extracted outcomes were procedural success, cardiac and non-cardiac death, MI, TLR, target vessel revascularization (TVR), ST, and MACE. Results were documented as count data and reported as percentages of the total study population. Due to significant study design heterogeneity between the included studies, a meta-analysis was not performed. Included articles were evaluated for potential biases using Cochrane risk of bias assessment tool (ROBINS-I) which was performed independently by two authors (RM, SN), with discrepancies adjudicated by a third author $(\mathrm{AD})$.

\section{Results}

Using the search strategy described above, we identified a total of 251 potentially relevances (Figure 1). Ninety-five duplicate publications were identified and removed. The remaining 156 references were screened. Non-English language articles, conference abstracts, review articles, editorials, case reports and case series with less than 10 patients were excluded. A total of 13 articles met the prespecified inclusion criteria for analysis.
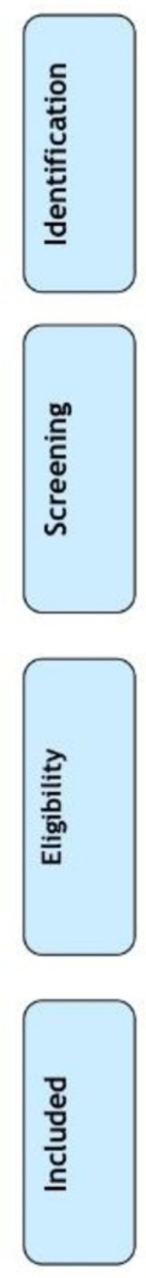

FIGURE 1: Study selection process flow diagram.
Records identified through database searching $(n=251)$ MEDLINE, $\mathrm{n}=52$ EMBASE, $\mathrm{n}=183$ Clinicaltrials.gov, $n=4$ Cochrane Library, $n=12$

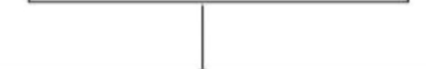

Records after duplicates removed ( $n=156$ )

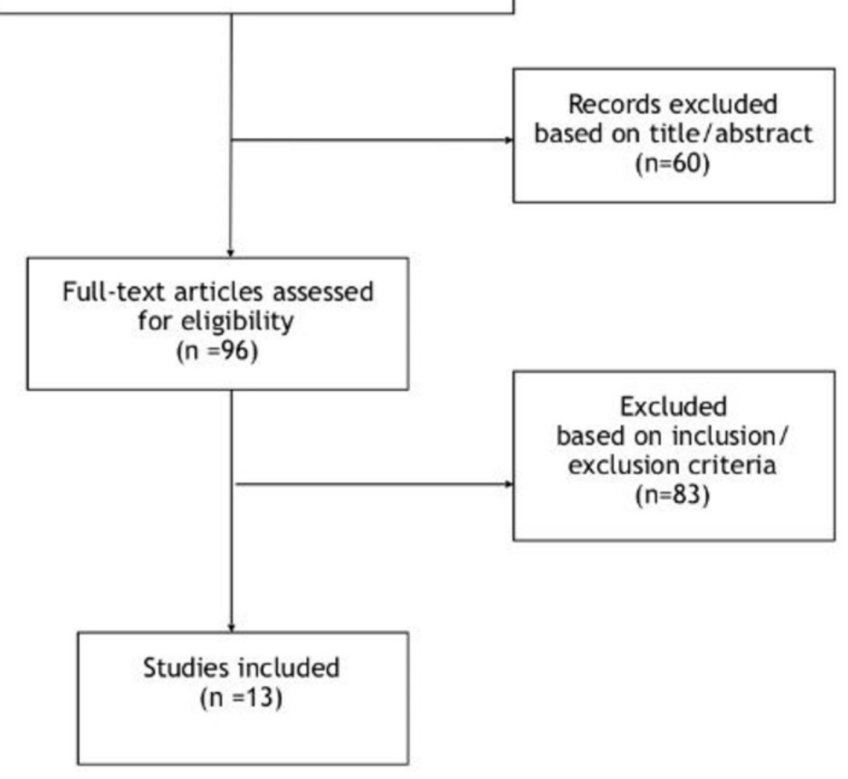




\section{Cureus}

\section{Study Characteristics}

All studies included into review were observational in nature and were published between 2014 and 2018. Data were reported for a total of 1,077 patients with median study population size of 41 (Interquartile range (IQR): 29.5-67.5) with number of patients ranging from 21 to 537. The median follow-up duration ranged from 108 to 703 days. Patients in the studies were predominantly male (77.5\%-98\%) with mean age ranging from 56.9 to 65.7 years. Eleven out of 13 publications were retrospective cohort studies and therefore did not include a control group. Two others were double arm studies which included comparator groups which retrospectively compared BVS with drug eluting stents (DES). All studies defined CTO similarly as $100 \%$ vessel occlusion with TIMI 0 flow for more than three months. Primary and secondary end points slightly varied from study to study (Table 1) but were mainly focused on individual or composite of major adverse cardiovascular events such as cardiac death, myocardial infarction, and ischemia-driven target lesion revascularization.

\begin{tabular}{|c|c|c|c|c|c|c|c|}
\hline $\begin{array}{l}\text { Author and } \\
\text { study title }\end{array}$ & $\begin{array}{l}\text { Year of } \\
\text { publication }\end{array}$ & $\begin{array}{l}\text { Study type and } \\
\text { design }\end{array}$ & $\begin{array}{l}\text { Number } \\
\text { of } \\
\text { patients }\end{array}$ & $\begin{array}{l}\text { Procedural } \\
\text { success }\end{array}$ & Primary outcomes & $\begin{array}{l}\text { Secondary } \\
\text { outcomes }\end{array}$ & $\begin{array}{l}\text { Follow-up } \\
\text { duration }\end{array}$ \\
\hline $\begin{array}{l}\text { La Manna } \\
\text { et al. [12] }\end{array}$ & 2018 & $\begin{array}{l}\text { Prospective } \\
\text { single center } \\
\text { series (GHOST- } \\
\text { CTO sub- } \\
\text { study) }\end{array}$ & 21 & $76.2 \%$ & $\begin{array}{l}\text { One-year optical coherence tomography } \\
\text { outcomes }\end{array}$ & $\begin{array}{l}\text { year defined as a } \\
\text { composite death, } \\
\text { MI, and TLR }\end{array}$ & $\begin{array}{l}\text { Median } 447 \\
\text { days (IQR } \\
365-713)\end{array}$ \\
\hline $\begin{array}{l}\text { Mitomo et } \\
\text { al. [13] }\end{array}$ & 2017 & $\begin{array}{l}\text { Retrospective } \\
\text { international } \\
\text { multicenter } \\
\text { registry }\end{array}$ & 65 & $100 \%$ & $\begin{array}{l}\text { Target lesion failure defined as a composite } \\
\text { cardiac death, target vessel MI, clinically driven } \\
\text { TLR }\end{array}$ & $\begin{array}{l}\text { All-cause } \\
\text { mortality, } \\
\text { clinically driven } \\
\text { target vessel } \\
\text { revascularization, } \\
\text { scaffold } \\
\text { thrombosis }\end{array}$ & $\begin{array}{l}\text { Median } 453 \\
\text { days }\end{array}$ \\
\hline $\begin{array}{l}\text { Maeremans } \\
\text { et al. [14] }\end{array}$ & 2017 & $\begin{array}{l}\text { Prospective } \\
\text { multicenter } \\
\text { single arm } \\
\text { study }\end{array}$ & 41 & $100 \%$ & $\begin{array}{l}\text { Incidence of TVF (in-stent re-stenosis or occlusion } \\
\text { with or without TVR) during FU period }\end{array}$ & $\begin{array}{l}\text { BVS patency and } \\
\text { performance of } \\
\text { quantitative } \\
\text { MSCT imaging } \\
\text { for determining } \\
\text { diameter and } \\
\text { area of stenosis } \\
\text { at one-year FU }\end{array}$ & 12 months \\
\hline $\begin{array}{l}\text { Kugler et } \\
\text { al. [15] }\end{array}$ & 2017 & $\begin{array}{l}\text { Retrospective } \\
\text { single center } \\
\text { study, two } \\
\text { arms, BVS } \\
\text { compared with } \\
\text { DES (patients } \\
\text { from Ulm-CSI } \\
\text { CTO study) }\end{array}$ & $\begin{array}{l}\text { BVS }=14 \\
\text { patients } \\
\text { with } 15 \\
\text { СTO, DES } \\
=15 \\
\text { patients }\end{array}$ & $\begin{array}{l}100 \% \text { for } \\
\text { both BVS } \\
\text { and DES } \\
\text { groups }\end{array}$ & $\begin{array}{l}\text { Composite cardiac death, MI not clearly related to } \\
\text { a nontarget vessel and target lesion } \\
\text { revascularization }\end{array}$ & N/A & $\begin{array}{l}\text { Angiographic } \\
\text { FU at } 9 \text { mo in } \\
96.7 \% \\
\text { lesions. } \\
\text { Clinical FU } \\
\text { at } 12 \mathrm{mo} \text { in } \\
100 \% \\
\text { patients }\end{array}$ \\
\hline $\begin{array}{l}\text { Fam et al. } \\
\text { [6] }\end{array}$ & 2017 & $\begin{array}{l}\text { Prospective } \\
\text { multicenter } \\
\text { single arm } \\
\text { registry }\end{array}$ & 105 & $97.1 \%$ & $\begin{array}{l}\text { Cardiac death, MI, scaftold thrombosis, clinically } \\
\text { driven TLR, non-TLR }\end{array}$ & N/A & Six months \\
\hline $\begin{array}{l}\text { Yamaç et } \\
\text { al. [16] }\end{array}$ & 2016 & $\begin{array}{l}\text { Prospective } \\
\text { single center } \\
\text { single arm } \\
\text { study }\end{array}$ & 30 & $100 \%$ & $\begin{array}{l}\text { All-cause mortality; cardiac death; and MACE } \\
\text { (non-fatal target vessel MI, TVR, symptom-driven } \\
\text { TLR, BVS thrombosis) }\end{array}$ & N/A & $\begin{array}{l}\text { Median } 542 \\
\text { days, (IQR } \\
\text { 175-961) }\end{array}$ \\
\hline $\begin{array}{l}\text { Vaquerizo } \\
\text { et al. [17] }\end{array}$ & 2016 & $\begin{array}{l}\text { Prospective } \\
\text { single arm } \\
\text { registry } \\
\text { (ABSORB-CTO } \\
\text { pilot study) }\end{array}$ & 33 & $100 \%$ & $\begin{array}{l}\text { Device patency investigated by multiple imagine } \\
\text { modalities }\end{array}$ & N/A & 12 months \\
\hline Ozel et al. & 2016 & $\begin{array}{l}\text { Prospective } \\
\text { single center }\end{array}$ & 41 & $100 \%$ & Rates of death, MI, angina, CABG, TLR, TVR & N/A & 12 months \\
\hline
\end{tabular}




\section{Cureus}

\begin{tabular}{|c|c|c|c|c|c|c|c|}
\hline \multicolumn{2}{|l|}{ [18] } & \multicolumn{6}{|l|}{$\begin{array}{l}\text { single arm } \\
\text { study }\end{array}$} \\
\hline $\begin{array}{l}\text { Lesiak et } \\
\text { al. [19] }\end{array}$ & 2016 & $\begin{array}{l}\text { Prospective } \\
\text { non- } \\
\text { randomized } \\
\text { clinical pilot } \\
\text { registry }\end{array}$ & 40 & $100 \%$ & $\begin{array}{l}\text { TVF, defined as the combination of cardiac death, } \\
\text { target vessel MI, or clinically driven TVR. Device } \\
\text { success (successful device deployment at the } \\
\text { intended segment), and procedure success rate } \\
\text { (residual stenosis }<30 \% \text { and TIMI flow grade } 3 \text {, } \\
\text { with no major procedural complications). }\end{array}$ & $\begin{array}{l}\text { Incidence of } \\
\text { scaffold } \\
\text { thrombosis }\end{array}$ & $\begin{array}{l}\text { Median } 556 \\
\text { days (274- } \\
932, \text { IQR } \\
374-706)\end{array}$ \\
\hline $\begin{array}{l}\text { Azzalini et } \\
\text { al. [20] }\end{array}$ & 2016 & $\begin{array}{l}\text { Retrospective } \\
\text { multicenter } \\
\text { registry }\end{array}$ & $\begin{array}{l}\text { BVS } \\
\text { group }(n= \\
\text { 153) was } \\
\text { compared } \\
\text { with DES } \\
\text { group ( } n= \\
384)\end{array}$ & $\begin{array}{l}99.3 \% \text { in } \\
\text { BVS } \\
\text { group }\end{array}$ & $\begin{array}{l}\text { Incidence of TVF, defined as the composite } \\
\text { cardiac death, target-vessel myocardial infarction, } \\
\text { and ischemia-driven TLR }\end{array}$ & N/A & $\begin{array}{l}\text { Median } 703 \\
\text { days (IQR } \\
426-989)\end{array}$ \\
\hline $\begin{array}{l}\text { Ojeda et al. } \\
\text { [21] }\end{array}$ & 2015 & $\begin{array}{l}\text { Single center } \\
\text { observational } \\
\text { study }\end{array}$ & 42 & $100 \%$ & $\begin{array}{l}\text { Technical success defined as patent vessel with } \\
<30 \% \text { residual stenosis and a TIMI flow grade } 3 \\
\text { achieved. MACE defined as a composite cardiac } \\
\text { death, MI, and TLR. Periprocedural MI. Scaffold } \\
\text { thrombosis. }\end{array}$ & N/A & $\begin{array}{l}\text { Mean: } 13 \pm 5 \\
\text { months, } \\
\text { median } 12 \\
\text { months (IQR } \\
9.75-16 \\
\text { months) }\end{array}$ \\
\hline $\begin{array}{l}\text { Goktekin et } \\
\text { al. [22] }\end{array}$ & 2015 & $\begin{array}{l}\text { Multicenter } \\
\text { registry }\end{array}$ & 70 & $100 \%$ & $\begin{array}{l}\text { Composite of all-cause death and non-fatal MI. } \\
\text { Composite safety endpoint of MACE, including } \\
\text { death, MI and symptom-driven TLR. }\end{array}$ & N/A & $\begin{array}{l}\text { Median } 11 \\
\text { months (IQR } \\
\text { 7-18 } \\
\text { months) }\end{array}$ \\
\hline $\begin{array}{l}\text { Wiebe et al. } \\
\text { [23] }\end{array}$ & 2014 & $\begin{array}{l}\text { Observational } \\
\text { study }\end{array}$ & 23 & $100 \%$ & $\begin{array}{l}\text { Procedural success defined as successful } \\
\text { deployment of the scaffold at the target lesion and } \\
\text { an estimated residual stenosis of } \leq 30 \% \text { on } \\
\text { angiography and optical coherence tomography. } \\
\text { MACE defined as cardiac death, MI, and } \\
\text { unscheduled percutaneous and surgical target } \\
\text { lesion. TVF included cardiac death, target vessel } \\
\mathrm{Ml} \text {, and percutaneous or surgical TVR. }\end{array}$ & N/A & $\begin{array}{l}\text { Median } 108 \\
\text { days }(79.5- \\
214.5)\end{array}$ \\
\hline
\end{tabular}

\section{TABLE 1: Overview of included studies.}

BVS: Bioresorbable vascular scaffolds; MSCT: Multi-slice computer tomography; FU: Follow-up; CTO: Chronic total occlusion; DES: Drug-eluting stents; MI: Myocardial infarction; MACE: Major adverse cardiovascular event; TVR: Target vessel revascularization; TVF: Target vessel failure; TLR: Target lesion revascularization; TIMI: Thrombolysis in myocardial infarction; IQR: Interquartile range; CABG: Coronary artery bypass grafting.

We identified only two double arm studies comparing BVS with DES that reported follow-up data (Table 2). The larger one was a multicenter retrospective registry which included a total of 537 patients by Azzalini et al. [20]. It compared outcomes for 153 patients who underwent first generation Absorb BVS implantation, and compared them to 384 patients treated with second generation DES. The primary endpoint was a target vessel failure (TVF) defined as the composite of cardiac death, target vessel myocardial infarction, and ischemia-driven TLR. Median follow-up duration was 703 (IQR: 426-989) days. There were no significant differences in rates of events between two groups including TVF (4.6\% vs 7.7\%; HR: 0.59, 95\% CI: 0.26-1.35; $\mathrm{p}=0.21$ ), ischemia-driven TLR (4.0\% vs $4.1 \%$; HR: 0.95 ; $95 \%$ CI: $0.37-2.45 ; \mathrm{p}=0.92)$, or scaffold or stent thrombosis $(0.6 \%$ vs $0.7 \%, p=0.86)$. Further inverse probability of treatment weight-adjusted Cox regression analysis still did not demonstrate any significant differences in outcomes between groups. However, when clinical, angiographic, and procedural variables were simultaneously added to the model, it showed a nonsignificant increase in risk of TVF and ischemia-driven TLR in the BVS group (adjusted HR: 3.45; 95\% CI: 0.87-13.66; $p=0.08$ ). Another double-arm observational study was performed by Kugler et al. [15]. It was a small prospective registry which included 29 patients among which 14 underwent BVS implantation and 15 received DES. Twelve-month clinical follow-up showed similar results to those reported by Azzalini et al. and did not demonstrate any difference in outcomes between BVS and DES groups. Risk of TVF and ischemia-driven TLR as well as scaffold thrombosis were comparable between two groups. 


\section{Cureus}

\begin{tabular}{|c|c|c|c|c|}
\hline Study & \multicolumn{2}{|c|}{ Kugler et al. [15] } & \multicolumn{2}{|c|}{ Azzalini et al. [20] } \\
\hline Study type & \multicolumn{2}{|c|}{ Single center retrospective study } & \multicolumn{2}{|c|}{ Multicenter retrospective registry } \\
\hline Arms & BVS & DES & BVS & DES \\
\hline \multicolumn{5}{|l|}{ Characteristics } \\
\hline $\mathrm{N}$ & 14 & 15 & 153 & 384 \\
\hline Mean age \pm SD/range, years & $60.5 \pm 7.8$ & $65.7 \pm 9.4$ & $60.0 \pm 9.3$ & $63.6 \pm 10.3$ \\
\hline Male, n (\%) & $12(85.7)$ & 14 (93.3) & $137(89.5)$ & $341(88.8)$ \\
\hline BMI, mean \pm SD & $27.8 \pm 3.7$ & $28.3 \pm 3.0$ & $28.4 \pm 5.0$ & $28.3 \pm 4.0$ \\
\hline Hypertension, n (\%) & $9(64.3)$ & $12(80.0)$ & $100(65.4)$ & $265(69.0)$ \\
\hline Dyslipidemia, n (\%) & $10(71.4)$ & $11(73.3)$ & $107(69.9)$ & $271(70.6)$ \\
\hline Diabetes, n (\%) & $2(14.3)$ & $2(13.3)$ & $52(34.0)$ & $134(34.9)$ \\
\hline Smoking, n (\%) & $8(57.1)$ & $10(66.7)$ & $38(24.8)$ & $79(20.6)$ \\
\hline CKD, n (\%) & $1(7.1)$ & $1(6.7)$ & $8(5.5)$ & $61(15.9)$ \\
\hline PAD, n (\%) & - & - & $12(8.3)$ & $86(22.4)$ \\
\hline Previous MI, n (\%) & - & - & $50(32.7)$ & $161(41.9)$ \\
\hline Previous PCl, n (\%) & - & - & $67(43.8)$ & 216 (56.3) \\
\hline Previous CABG, n (\%) & $0(0.0)$ & $2(13.3)$ & $4(2.6)$ & $38(9.9)$ \\
\hline Previous TIA/stroke, n (\%) & - & - & $4(2.6)$ & $34(8.9)$ \\
\hline \multicolumn{5}{|l|}{ Outcomes } \\
\hline $\mathrm{N}$ & 15 lesions & 15 & 151 & 363 \\
\hline Procedural success rate, \% & - & - & 99.3 & 96.6 \\
\hline DOCE/MACE & $1(6.7)$ & $2(13.3)$ & $7(4.6)$ & $28(7.7)$ \\
\hline Cardiac death & $0(0.0)$ & $0(0.0)$ & $2(1.3)$ & $11(3.0)$ \\
\hline Non-cardiac death & - & - & - & - \\
\hline TLR & $1(6.7)$ & $2(13.3)$ & $6(4.0)$ & $15(4.1)$ \\
\hline MI & $0(0.0)$ & $0(0.0)$ & - & - \\
\hline TV MI & $0(0.0)$ & $0(0.0)$ & $1(0.7)$ & $5(1.4)$ \\
\hline TVR & - & - & - & - \\
\hline TLF/TVF & $1(6.7) /-$ & $2(13.3) /-$ & $-/ 7(4.6)$ & $-/ 28(7.7)$ \\
\hline ST & $0(0.0)$ & $0(0.0)$ & $1(0.7)$ & $2(0.6)$ \\
\hline Restenosis & $1(6.7)$ & $2(13.3)$ & $6(3.97)$ & $15(4.13)$ \\
\hline
\end{tabular}

\section{TABLE 2: Baseline patient characteristics and outcomes for double arm studies.}

BMI: Body mass index; CABG: Coronary artery bypass grafting; CKD: Chronic kidney disease; DOCE: Device-oriented composite endpoint; MACE: Major adverse cardiac events; MI: Myocardial infarction; PAD: Peripheral artery disease; PCI: Percutaneous coronary intervention; TIA: Transient ischemic attack; TLR: Target lesion revascularization; TV: Target vessel; TVR: Target vessel revascularization; TVF: Target vessel failure; SD: Standard deviation; ST: Scaffold/stent thrombosis.

Single Arm Studies

This group includes 11 publications among which the majority were small retrospective and prospective registries [6, 12-14, 16-19, 21-23]. Patient demographic and clinical characteristics were similar in all studies 


\section{Cureus}

with similar reported outcomes (Table 3).

\begin{tabular}{|c|c|c|c|c|c|c|c|c|c|c|c|}
\hline Study & $\begin{array}{l}\text { La Manna et } \\
\text { al. }[12]\end{array}$ & Mitomo et al. [13] & $\begin{array}{l}\text { Maeremans } \\
\text { et al. [14] }\end{array}$ & Fam et al. [6] & $\begin{array}{l}\text { Yamaç et al. } \\
\text { [16] }\end{array}$ & $\begin{array}{l}\text { Vaquerizo } \\
\text { et al. [17] }\end{array}$ & $\begin{array}{l}\text { Özel et al. } \\
{[18]}\end{array}$ & Lesiak et al. [19] & $\begin{array}{l}\text { Ojeda et al. } \\
\text { [21] }\end{array}$ & $\begin{array}{l}\text { Goktekin et } \\
\text { al. [22] }\end{array}$ & $\begin{array}{l}\text { Wiebe et al. } \\
{[23]}\end{array}$ \\
\hline Study type & $\begin{array}{l}\text { Prospective } \\
\text { single center } \\
\text { series }\end{array}$ & $\begin{array}{l}\text { International } \\
\text { multicenter } \\
\text { retrospective registry }\end{array}$ & $\begin{array}{l}\text { Multicenter } \\
\text { prospective } \\
\text { study }\end{array}$ & $\begin{array}{l}\text { Multicenter } \\
\text { prospective } \\
\text { registry }\end{array}$ & $\begin{array}{l}\text { Single center } \\
\text { prospective } \\
\text { study }\end{array}$ & $\begin{array}{l}\text { Prospective } \\
\text { registry }\end{array}$ & $\begin{array}{l}\text { Single center } \\
\text { prospective } \\
\text { study }\end{array}$ & $\begin{array}{l}\text { Prospective, non- } \\
\text { randomized clinical } \\
\text { pilot registry }\end{array}$ & $\begin{array}{l}\text { Single center } \\
\text { observational } \\
\text { study }\end{array}$ & $\begin{array}{l}\text { Multicenter } \\
\text { prospective } \\
\text { registry }\end{array}$ & $\begin{array}{l}\text { Multicenter } \\
\text { observational } \\
\text { study }\end{array}$ \\
\hline \multicolumn{12}{|l|}{ Characteristics } \\
\hline $\mathrm{N}$ & 21 & 65 & 41 & 105 & 30 & 33 & 41 & 40 & 42 & 70 & 23 \\
\hline $\begin{array}{l}\text { Mean age } \pm \\
\text { SD/range, } \\
\text { years }\end{array}$ & $62.19 \pm 7.9$ & $60.8 \pm 11.0$ & $60.0 \pm 11.0$ & $59.4 \pm 8.96$ & $57.8 \pm 9.6$ & $61 \pm 10$ & $61.9 \pm 9.7$ & $59.9 \pm 8.3$ & $58.0 \pm 9.0$ & $56.9 \pm 9.4$ & $60.4 \pm 9.0$ \\
\hline Male, n (\%) & $17(81)$ & $58(89.2)$ & $34(83)$ & $94(89.5)$ & 26 (86.7) & $28(80)$ & 35 (85.4) & 31 (77.5) & $41(98)$ & $63(90.0)$ & $19(82.6)$ \\
\hline $\begin{array}{l}\text { BMI, mean } \pm \\
\text { SD }\end{array}$ & $28.4 \pm 3.9$ & - & $29 \pm 4.8$ & - & - & - & - & - & - & - & $27.8 \pm 3.9$ \\
\hline $\begin{array}{l}\text { Hypertension, } \\
\text { n (\%) }\end{array}$ & $17(81)$ & $44(67.8)$ & $30(73)$ & $73(69.5)$ & $24(80.0)$ & - & $33(80.5)$ & $32(80.0)$ & $24(57)$ & 55 (78.6) & $21(91.3)$ \\
\hline $\begin{array}{l}\text { Dyslipidemia, n } \\
\text { (\%) }\end{array}$ & $16(76.2)$ & $40(61.5)$ & $30(73)$ & 76 (72.4) & $17(56.7)$ & - & $19(46.3)$ & - & $27(64)$ & $37(52.9)$ & $15(65.2)$ \\
\hline $\begin{array}{l}\text { Diabetes, n } \\
(\%)\end{array}$ & $9(42.8)$ & 26 (40.0) & $12(29)$ & $35(33.3)$ & $1(3.3)$ & $7(20.0)$ & $21(51.2)$ & $12(30.0)$ & $14(33)$ & 15 (21.4) & $8(34.8)$ \\
\hline Smoking, $\mathrm{n}(\%)$ & $8(38.1)$ & - & 9 (22) & $51(48.6)$ & $12(40.0)$ & - & $14(34.1)$ & $14(35.0)$ & $8(19)$ & $12(17.1)$ & $11(47.8)$ \\
\hline CKD, n (\%) & - & 26 (40.1) & - & - & $0(0.0)$ & - & - & $6(15.0)$ & - & $2(2.9)$ & - \\
\hline PAD, n (\%) & $1(4.8)$ & $6(9.2)$ & $3(7)$ & - & - & - & - & - & - & - & - \\
\hline $\begin{array}{l}\text { Previous MI, n } \\
\text { (\%) }\end{array}$ & $7(33.3)$ & $11(16.9)$ & $10(24)$ & 31 (29.5) & $3(10.0)$ & $\cdot$ & $27(65.9)$ & $20(50.0)$ & $12(28)$ & $6(8.6)$ & - \\
\hline $\begin{array}{l}\text { Previous PCl, } \\
\text { n (\%) }\end{array}$ & $17(81)$ & 35 (53.8) & 11 (27) & $49(46.7)$ & $4(13.3)$ & $13(37)$ & $23(56.1)$ & $18(45.0)$ & $15(36)$ & $12(17.1)$ & - \\
\hline $\begin{array}{l}\text { Previous } \\
\text { CABG, n (\%) }\end{array}$ & 0 & $4(6.2)$ & $3(7)$ & $3(2.9)$ & $2(6.7)$ & - & $7(17.1)$ & $2(5.0)$ & - & $7(10.0)$ & - \\
\hline $\begin{array}{l}\text { Previous } \\
\text { TIA/stroke, n } \\
(\%)\end{array}$ & $1(4.8)$ & $20(30.8)$ & $2(5)$ & - & (0.0) & - & - & - & - & $0(0.0)$ & - \\
\hline Outcomes & & & & & & & & & & & \\
\hline $\begin{array}{l}\text { Procedural } \\
\text { success rate, } \\
\%\end{array}$ & 76.2 & - & - & 97.1 & - & 100 & - & - & 98 & - & - \\
\hline DOCE/MACE & $1(1.2)$ & $0(0.0)$ & - & - & - & - & - & - & $2(4.8)$ & $2(2.9)$ & $1(4.3)$ \\
\hline Cardiac death & 0 & $0(0.0)$ & $0(0.0)$ & $0(0.0)$ & $1(3.0)$ & $0(0.0)$ & $0(0.0)$ & $0(0.0)$ & $0(0.0)$ & $0(0.0)$ & $0(0.0)$ \\
\hline $\begin{array}{l}\text { Non-cardiac } \\
\text { death }\end{array}$ & 0 & $0(0.0)$ & $1(2.4)$ & $0(0.0)$ & $0(0.0)$ & - & $0(0.0)$ & $0(0.0)$ & $0(0.0)$ & $0(0.0)$ & $0(0.0)$ \\
\hline TLR & 0 & $0(0.0)$ & $0(0.0)$ & 2/96 (2.1) & $3(8.6)$ & $0(0.0)$ & $1(2.4)$ & - & $2(4.8)$ & $2(2.9)$ & $1(4.3)$ \\
\hline MI & $1(1.2)$ & $0(0.0)$ & - & 2/96 (2.1) & $0(0.0)$ & $0(0.0)$ & $1(2.4)$ & $2(5.0)$ & $0(0.0)$ & $0(0.0)$ & $0(0.0)$ \\
\hline TV MI & 0 & $0(0.0)$ & - & - & $0(0.0)$ & $0(0.0)$ & $1(2.4)$ & $2(5.0)$ & $0(0.0)$ & $0(0.0)$ & $0(0.0)$ \\
\hline TVR & 0 & $4(6.2)$ & $0(0.0)$ & - & $5(14.3)$ & $0(0.0)$ & $5(12.2)$ & $3(7.5)$ & - & $1(1.4)$ & \\
\hline TVF & 0 & - & $0(0.0)$ & - & & $0(0.0)$ & - & $3(7.5)$ & - & - & $1(4.3)$ \\
\hline ST & 0 & $0(0.0)$ & - & $1 / 96$ & $0(0.0)$ & 0.0 & - & $(5.0)$ & $\begin{array}{l}0(0.0) \\
0\end{array}$ & 0 & $1(4.3)$ \\
\hline
\end{tabular}




\section{Cureus}

Restenosis 0

2/96 (2.1)

$3(8.6)$

$2(6.0)$

$3(7.1)$

$2(2.9)$

\section{TABLE 3: Baseline patient characteristics and outcomes for single arm studies.}

BMI: Body mass index; CABG: Coronary artery bypass grafting; CKD: Chronic kidney disease; DOCE: Device-oriented composite endpoint; MACE: Major adverse cardiac events; MI: Myocardial infarction; PAD: Peripheral artery disease; PCI: Percutaneous coronary intervention; TIA: Transient ischemic attack; TLR: Target lesion revascularization; TV: Target vessel; TVR: Target vessel revascularization; TVF: Target vessel failure; SD: Standard deviation; ST: Scaffold/stent thrombosis.

Median follow-up duration in this group varied from 108 to 556 days. A composite of cardiac death, target vessel MI, and target lesion revascularization was reported for five out of 11 studies and was $2.64 \%$ (95\% CI: $1.04 \%$ to $4.24 \%$ ). Only one study, performed by Yamac et al., reported a single cardiac death (3\% of their total population) during the follow-up period [16]. Maeremans et al. were the only authors to report a noncardiac death (2.4\% of their study population) [14]. Incidence of non-fatal myocardial infarction was $1.07 \%$ ( $95 \%$ CI: $0.09 \%$ to $2.06 \%$ ). Incidence of target lesion revascularization was $2.51 \%$ (95\% CI: $0.86 \%$ to $4.16 \%$ ) with the highest rate of $8.6 \%$ reported by Yamac et al. [16]. The incidence of probable or definite scaffold thrombosis was $1.3 \%$ ( $95 \%$ CI: $-0.09 \%$ to $2.39 \%$ ). Composite rate of restenosis reported in six studies was $4.45 \%$ (95\% CI: $2.04 \%$ to $6.86 \%$ ). Using ROBINS-I assessment tool for non-randomized studies, each publication underwent a thorough evaluation for potential risk of bias which demonstrated that 12 out of 13 studies had overall critical bias risk since at least one of the assessed domains in each study was judged as having critical bias risk. The double arm study published by Azzalini et al. was judged as having serious risk of bias (Table 4). 


\begin{tabular}{|c|c|c|c|c|c|c|c|c|}
\hline $\begin{array}{l}\text { Study/author } \\
\text { name }\end{array}$ & $\begin{array}{l}\text { Bias } d / t \\
\text { confounding }\end{array}$ & $\begin{array}{l}\text { Bias in } \\
\text { selection } \\
\text { participants }\end{array}$ & $\begin{array}{l}\text { Bias in } \\
\text { classification of } \\
\text { interventions }\end{array}$ & $\begin{array}{l}\text { Bias } d / t \text { deviations } \\
\text { from intended } \\
\text { intervention }\end{array}$ & $\begin{array}{l}\text { Bias } d / t \\
\text { missing } \\
\text { data }\end{array}$ & $\begin{array}{l}\text { Bias in } \\
\text { measurement of } \\
\text { outcomes }\end{array}$ & $\begin{array}{l}\text { Bias in selection } \\
\text { of reported } \\
\text { results }\end{array}$ & $\begin{array}{l}\text { Overall } \\
\text { bias }\end{array}$ \\
\hline $\begin{array}{l}\text { La Manna et } \\
\text { al. [12] }\end{array}$ & Critical & Critical & Serious & Moderate & Serious & Critical & Moderate & Critical \\
\hline $\begin{array}{l}\text { Mitomo et al. } \\
\text { [13] }\end{array}$ & Critical & Serious & Critical & Moderate & Critical & Critical & Moderate & Critical \\
\hline $\begin{array}{l}\text { Maeremans } \\
\text { et al. [14] }\end{array}$ & Critical & Critical & Serious & Moderate & Serious & Critical & Moderate & Critical \\
\hline $\begin{array}{l}\text { Kugler et al. } \\
\text { [15] }\end{array}$ & Critical & Serious & Critical & Moderate & Critical & Critical & Moderate & Critical \\
\hline Fam et al. [6] & Critical & Critical & Serious & Moderate & Moderate & Critical & Moderate & Critical \\
\hline $\begin{array}{l}\text { Yamaç et al. } \\
\text { [16] }\end{array}$ & Critical & Critical & Serious & Moderate & Moderate & Critical & Moderate & Critical \\
\hline $\begin{array}{l}\text { Vaquerizo et } \\
\text { al. [17] }\end{array}$ & Critical & Critical & Serious & Moderate & Moderate & Critical & Moderate & Critical \\
\hline $\begin{array}{l}\text { Ozel et al. } \\
\text { [18] }\end{array}$ & Critical & Critical & Serious & Moderate & Moderate & Critical & Moderate & Critical \\
\hline $\begin{array}{l}\text { Lesiak et al. } \\
\text { [19] }\end{array}$ & Critical & Critical & Serious & Moderate & Moderate & Critical & Moderate & Critical \\
\hline $\begin{array}{l}\text { Azzalinı et al. } \\
\text { [20] }\end{array}$ & Serious & Serious & Serious & Moderate & Moderate & Serious & Moderate & Serious \\
\hline $\begin{array}{l}\text { Ojeda et al. } \\
\text { [21] }\end{array}$ & Critical & Critical & Serious & Moderate & Moderate & Critical & Moderate & Critical \\
\hline $\begin{array}{l}\text { Goktekin et } \\
\text { al. [22] }\end{array}$ & Critical & Critical & Serious & Moderate & Moderate & Critical & Moderate & Critical \\
\hline $\begin{array}{l}\text { [23] } \\
\text { [2]et et al. }\end{array}$ & Critical & Critical & Serious & Moderate & Moderate & Critical & Moderate & Critical \\
\hline
\end{tabular}

TABLE 4: Individual study evaluation for risk of bias using Cochrane ROBINS-I tool.

\section{Discussion}

Although DES remain devices of choice for percutaneous coronary interventions, BVS were designed as an alternative to DES with the theoretical advantages of full resorption of scaffolds after two years with resultant restoration of vasomotion, potentially avoiding the "caged vessel" phenomena leading to late stent thrombosis $[9,10]$. Those properties in theory should be especially advantageous in CTO revascularization procedures where long segment stenting is often required. However, these theoretical advantages would need to be proven in trials before widespread adoption of this new technology. Our review identified 13 studies of overall low-quality evidence. The review of included articles showed favorable mid- to long-term outcomes for BVS implantation in CTO. Most of the studies showed relatively low incidence of composite as well as individual end points after a fairly long follow-up period. One large double-arm study performed by Azzalini et al. did not demonstrate any statistically significant difference in long-term outcomes between DES and BVS groups in unadjusted and primary adjusted analysis but showed some tendency toward a higher adjusted risk of ischemia-driven target lesion failure in BVS group compared with DES [20]. In contrast to the lower-quality evidence we have complied in CTO, three new randomized trials have identified an increased risk of stent thrombosis when compared to drug-eluting stents for patients undergoing routine PCI [24-26]. The differences in our findings from these RCTs might be explained by differing hemodynamics of stenting of CTOs, but could also be explained by patient selection, limited followup and reporting differences in our less rigorous study designs in this review. This trend might also be explained by comparison of first generation of BVS with second generation of DES, which have much thinner struts and as a result creates less turbulence of blood flow with less risk of thrombosis and restenosis [27]. As a result of described rheological disturbances, recently raised concerns about increased scaffold thrombosis (ST) were confirmed by several clinical trials and recently published meta-analysis which showed increased incidence of scaffold thrombosis compared with DES [24-26]. Interestingly, our 
systematic review did not demonstrate any difference in thrombosis rate between BVS and DES in CTO lesions and even in single arm observational studies thrombosis incidence was low. This again may be related to relatively small sample size but can also be explained by meticulous lesion selection for intervention, avoiding of small vessels with lower blood flow velocities, use of additional imaging modalities such as intravascular ultrasound or optical coherence tomography, more experienced performing operators given the complexity of CTO lesions. Lastly, recent concerns for potential subclinical nature of CTO lesion restenosis and even stent/scaffold thrombosis should also be considered [20]. For this reason, angiographic follow-up is important to uncover true restenosis/scaffold thrombosis incidence rates. In our systematic review, eight out of 12 studies performed an actual invasive or non-invasive follow-up angiography after BVS implantation, but in the vast majority of those studies, this imaging was done in less than $100 \%$ of patients, so the true rates of restenosis or scaffold thrombosis might be underestimated. For instance, Mitomo et al. reported that only 33.8\% of patients underwent follow-up angiography [13] while Maeremans et al. reported about $83 \%$ of patients having follow-up multislice computed tomography angiography [14]. Regardless of these promising findings, the first generation BVS was recently removed from clinical practice due to safety alert for Absorb BVS (Abbott) recently issued by Food and Drug Administration due to confirmed higher rates of ST [28]. Next generation of BVS with thinner struts and improved rheological parameters are currently under investigation with some promising preliminary results [29]. At the same time, there are no published data on the use of second-generation scaffolds in CTO yet. Our systematic review has several limitations. First, all included studies were observational in nature with no published randomized controlled trials available to date and thus, the available data are subject to potential biases, such as selection bias and confounding, and as such were deemed to be of low quality in our quality review. Second, due to significant study heterogeneity, we were unable to meta-analyze our data across included publications and therefore we instead conducted qualitative systematic review. Third, median follow-up duration was less than two years for most of the studies, which limits the ability to assess theoretical BVS advantages after scaffold resorption. Fourth, all studies used first-generation BVS Absorb with its potentially unfavorable rheological properties.

\section{Conclusions}

Although data on the use of first-generation BVS in CTO are sporadic and limited by small sample observational studies, available evidence to date is promising and suggests of acceptable mid- and long-term outcomes comparable with second generation DES. Further investigation with randomized clinical trials and use of better-designed newer generation scaffolds is required in order to control for confounding variables and to determine if there is a significant difference between these devices.

\section{Appendices}

\section{Search strategy for PubMed}

("bioresorbable vascular" OR "bioresorbable scaffold" OR "bioresorbable scaffolds" OR "bioresorbable stent" OR "bioresorbable vascular scaffolds" OR "absorb bioresorbable vascular scaffold" OR "bioresorbable coronary scaffold" OR "bioresorbable scaffolds coronary" OR "BVS" OR "BRS" OR "naturally dissolving stents") AND ("chronic total occlusion" OR "chronic total occlusions" OR "coronary chronic total occlusion" OR "CTO" OR "chronic total coronary occlusion").

\section{Search strategy for Embase}

('bioresorbable scaffold'/exp OR 'absorb (device)' OR 'absorb gt1' OR 'acute (device)' OR 'amaranth (device)' OR 'avatar (device)' OR 'desolve' OR 'dreams (device)' OR 'fortitude (device)' OR 'fantom (device)' OR 'ideal biostent' OR 'meres (device)' OR 'rezolve' OR 'xinsorb' OR 'zorion' OR 'bioresorbable scaffold' OR 'bioresorbable vascular stent'/exp OR 'igaki-tamai' OR 'bioresorbable vascular stent' OR 'bvs' OR 'brs') AND ('chronic total occlusion'/exp OR 'chronic total occlusion' OR 'chronic total coronary occlusion'/exp OR 'chronic total coronary artery occlusion'/exp OR 'chronic total occlusion percutaneous coronary intervention'/exp OR 'cto').

\section{Additional Information \\ Disclosures}

Conflicts of interest: In compliance with the ICMJE uniform disclosure form, all authors declare the following: Payment/services info: All authors have declared that no financial support was received from any organization for the submitted work. Financial relationships: All authors have declared that they have no financial relationships at present or within the previous three years with any organizations that might have an interest in the submitted work. Other relationships: All authors have declared that there are no other relationships or activities that could appear to have influenced the submitted work.

\section{References}

1. Di Mario C, Werner GS, Sianos G, et al.: European perspective in the recanalisation of Chronic Total Occlusions (CTO): consensus document from the EuroCTO Club. EuroIntervention. 2007, 3:30-43.

2. Fefer P, Knudtson ML, Cheema AN, et al.: Current perspectives on coronary chronic total occlusions: the 
Canadian Multicenter Chronic Total Occlusions Registry. J Am Coll Cardiol. 2012, 59:991-997.

10.1016/j.jacc.2011.12.007

3. Christakopoulos GE, Christopoulos G, Carlino M, et al.: Meta-analysis of clinical outcomes of patients who underwent percutaneous coronary interventions for chronic total occlusions. Am J Cardiol. 2015, 115:13671375. 10.1016/j.amjcard.2015.02.038

4. Chung C-M, Nakamura S, Tanaka K, et al.: Effect of recanalization of chronic total occlusions on global and regional left ventricular function in patients with or without previous myocardial infarction. Catheter Cardiovasc Interv. 2003, 60:368-374. 10.1002/ccd.10641

5. Jang WJ, Yang JH, Choi S-H, et al.: Long-term survival benefit of revascularization compared with medical therapy in patients with coronary chronic total occlusion and well-developed collateral circulation. JACC Cardiovasc Interv. 2015, 8:271-279. 10.1016/j.jcin.2014.10.010

6. Fam JM, Ojeda S, Garbo R, et al.: Everolimus-eluting bioresorbable vascular scaffolds for treatment of complex chronic total occlusions. EuroIntervention. 2017, 13:355-363. 10.4244/EIJ-D-16-00253

7. Suh J, Park D-W, Lee J-Y, et al.: The relationship and threshold of stent length with regard to risk of stent thrombosis after drug-eluting stent implantation. JACC Cardiovasc Interv. 2010, 3:383-389. 10.1016/j.jcin.2009.10.033

8. Shirai S, Kimura T, Nobuyoshi M, et al.: Impact of multiple and long sirolimus-eluting stent implantation on 3-year clinical outcomes in the j-Cypher Registry. JACC Cardiovasc Interv. 2010, 3:180-188. 10.1016/j.jcin.2009.11.009

9. Serruys PW, Ormiston JA, Onuma Y, et al.: A bioabsorbable everolimus-eluting coronary stent system (ABSORB): 2-year outcomes and results from multiple imaging methods. Lancet. 2009, 373:897-910. 10.1016/S0140-6736(09)60325-1

10. Serruys PW, Ormiston J, Van Geuns R-J, et al.: A polylactide bioresorbable scaffold eluting everolimus for treatment of coronary stenosis: 5-year follow-up. J Am Coll Cardiol. 2016, 67:766-776. 10.1016/j.jacc.2015.11.060

11. Liberati A, Altman DG, Tetzlaff J, et al.: The PRISMA statement for reporting systematic reviews and metaanalyses of studies that evaluate health care interventions: explanation and elaboration. J Clin Epidemiol. 2009, 62:1-34. 10.1016/j.jclinepi.2009.06.006

12. La Manna A, Miccichè E, D'Agosta G, et al.: Vascular response and healing profile of everolimus-eluting bioresorbable vascular scaffolds for percutaneous treatment of chronic total coronary occlusions: a oneyear optical coherence tomography analysis from the GHOST-CTO registry. Int J Cardiol. 2018, 253:45-49. 10.1016/j.ijcard.2017.10.107

13. Mitomo S, Naganuma T, Fujino Y, et al.: Bioresorbable vascular scaffolds for the treatment of chronic total occlusions: an international multicenter registry. Circ Cardiovasc Interv. 2017, 10:e004265. 10.1161/CIRCINTERVENTIONS.116.004265

14. Maeremans J, Verhaert D, Pereira B, et al.: One-year clinical and computed tomography follow-up after implantation of bioresorbable vascular scaffolds in patients with coronary chronic total occlusions. Catheter Cardiovasc Interv. 2018, 92:488-496. 10.1002/ccd.27390

15. Kugler C, Marovic S, Rottbauer W, Wöhrle J: Bioresorbable scaffolds compared with everolimus-eluting stents for the treatment of chronic coronary total occlusion: clinical and angiographic results of a matched paired comparison. Coronary Artery Disease. 2017, 28:120-125. 10.1097/MCA.0000000000000449

16. Yamac AH, Yildiz A, Nasifov M, Tastan A, Bashirov N, Goktekin O: Clinical and angiographic outcomes at more than 1 year after treatment of chronic total occlusions with the everolimus-eluting bioresorbable vascular scaffold. Turk Kardiyol Dern Ars. 2016, 44:647-655. 10.5543/tkda.2016.21774

17. Vaquerizo B, Barros A, Pujadas S, et al.: One-year results of bioresorbable vascular scaffolds for coronary chronic total occlusions. Am J Cardiol. 2016, 117:906-917. 10.1016/j.amjcard.2015.12.025

18. Özel E, Taştan A, Öztürk A, Özcan EE, Kilicaslan B, Özdogan O: Procedural and one-year clinical outcomes of bioresorbable vascular scaffolds for the treatment of chronic total occlusions: a single-centre experience. Cardiovasc J Afr. 2016, 27:345-349. 10.5830/CVJA-2016-033

19. Lesiak M, Lanocha M, Araszkiewicz A, et al.: Percutaneous coronary intervention for chronic total occlusion of the coronary artery with the implantation of bioresorbable everolimus-eluting scaffolds. Poznan CTOAbsorb Pilot Registry. EuroIntervention. 2016, 12:144-151. 10.4244/EIJV12I2A27

20. Azzalini L, Giustino G, Ojeda S, et al.: Procedural and long-term outcomes of bioresorbable scaffolds versus drug-eluting stents in chronic total occlusions: the BONITO registry (bioresorbable scaffolds versus drugeluting stents in chronic total occlusions). Circ Cardiovasc Interv. 2016, 9:e004284. 10.1161/CIRCINTERVENTIONS.116.004284

21. Ojeda S, Pan M, Romero M, et al.: Outcomes and computed tomography scan follow-up of bioresorbable vascular scaffold for the percutaneous treatment of chronic total coronary artery occlusion. Am J Cardiol. 2015, 115:1487-1493. 10.1016/j.amjcard.2015.02.048

22. Goktekin O, Yamac AH, Latib A, et al.: Evaluation of the safety of everolimus-eluting bioresorbable vascular scaffold (BVS) implantation in patients with chronic total coronary occlusions: acute procedural and shortterm clinical results. J Invasive Cardiol. 2015, 27:461-466.

23. Wiebe J, Liebetrau C, Dörr O, et al.: Feasibility of everolimus-eluting bioresorbable vascular scaffolds in patients with chronic total occlusion. Int J Cardiol. 2015, 179:90-94. 10.1016/j.ijcard.2014.10.032

24. Serruys PW, Chevalier B, Sotomi Y, et al.: Comparison of an everolimus-eluting bioresorbable scaffold with an everolimus-eluting metallic stent for the treatment of coronary artery stenosis (ABSORB II): a 3 year, randomised, controlled, single-blind, multicentre clinical trial. Lancet. 2016, 388:2479-2491. 10.1016/S0140-6736(16)32050-5

25. Wykrzykowska JJ, Kraak RP, Hofma SH, et al.: Bioresorbable scaffolds versus metallic stents in routine PCI . N Engl J Med. 2017, 376:2319-2328. 10.1056/NEJMoa1614954

26. Cassese S, Byrne RA, Ndrepepa G, et al.: Everolimus-eluting bioresorbable vascular scaffolds versus everolimus-eluting metallic stents: a meta-analysis of randomised controlled trials. Lancet. 2016, 387:537544. 10.1016/S0140-6736(15)00979-4

27. Pache J, Kastrati A, Mehilli J, et al.: Intracoronary stenting and angiographic results: strut thickness effect 


\section{Cureus}

on restenosis outcome (ISAR-STEREO-2) trial. J Am Coll Cardiol. 2003, 41:1283-1288. 10.1016/S0735-

1097(03)00119-0

28. UPDATE on increased rate of major adverse cardiac events observed in patients receiving Abbott Vascular's Absorb GT1 bioresorbable vascular scaffold (BVS) - Letter to health care providers. (2017). Accessed: February 15, 2018:

https://www.fda.gov/MedicalDevices/Safety/LetterstoHealthCareProviders/ucm582728.htm.

29. FUTURE-I: six-month clinical, angiographic IVUS, and OCT results with a thin-strut PLLA-based sirolimuseluting bioresorbable vascular scaffold in patients with coronary artery disease. (2016). Accessed: March 04, 2018: https://www.tctmd.com/videos/futurei-sixmonth-clinical-angiographic-ivus-and-oct-results-with-athinstrut-pllabased-s.... 